\title{
Avaliação do surgimento de comorbidades em pacientes com doença de Graves tratados com iodo radioativo em acompanhamento por mais de 10 anos
}

\author{
Emerging comorbidities in Graves' disease patients treated \\ with radioiodine with more than 10 years of follow-up
}

Fernanda Vieira Ramalho de Azevedo', Francisco Gomes da Silva Blotta', Juliana Malheiros Goirgetta', Rosângela Noé², Mário Vaisman'

\section{RESUMO}

Objetivos: Avaliar o surgimento de comorbidades cardiovasculares e/ou neoplásicas e a taxa de mortalidade nos pacientes com hipertireoidismo em decorrência da doença de Graves tratados com iodo radioativo há mais de 10 anos. Materiais e métodos: Estudo retrospectivo com análise de prontuários do Hospital Universitário Clementino Fraga Filho, Universidade Federal do Rio de Janeiro, entre janeiro de 1981 e novembro de 1999. Resultados: Foram avaliados 107 pacientes (93 mulheres e 14 homens), com uma mediana de idade de 54 anos. Comparando o grupo de pacientes que receberam iodo radiativo com grupo de pacientes eutireoidianos pós-tratamento com drogas antitireoidianas (DAT), foi observado aumento significativo no surgimento de hipertensão arterial (HAS) e dislipidemia, mas não na taxa de mortalidade. Conclusão: Para avaliar a real influência da terapêutica com iodo radioativo no surgimento dessas comorbidades e na taxa de mortalidade, é necessário um tempo maior de acompanhamento. A idade e o tempo de exposição aos efeitos do hipertireoidismo parecem influenciar no surgimento dessas comorbidades. Arq Bras Endocrinol Metab. 2013;57(1):51-6

Descritores

lodo radioativo; hipertireoidismo; comorbidades; mortalidade

\begin{abstract}
Objectives: To evaluate the occurrence of cardiovascular disease and malignant tumors and the mortality rate in patients who received radioiodine treatment for hyperthyroidism due to Grave's disease with at least ten years of follow-up. Materials and methods: The medical records of all patients who were treated with ${ }^{131}$ for Graves' disease at Hospital Universitário Clementino Fraga Filho, Universidade Federal do Rio de Janeiro, were reviewed retrospectively, between January, 1981 and November, 1999. Results: Data from 107 patients (14 men and 93 women), with median age of 54 years were analyzed. Comparing the group of patients who were treated with $\mathrm{I}^{131}$ therapy with a group of euthyroid patients post-treatment with antithyroid drugs, a significant increase in the occurrence of hypertension and dyslipidemia was observed, but not in mortality rate. Conclusion: To evaluate the real influence of the treatment with radioactive iodine in the occurrence of these comorbidities and the mortality rate, we need a longer follow-up. The age and time of exposure to the effects of hyperthyroidism seem to influence the occurrence of these comorbidities. Arq Bras Endocrinol Metab. 2013;57(1):51-6
\end{abstract}

Keywords

Radioiodine; hyperthyroidism; comorbidities; mortality

Correspondência para: Fernanda Vieira Ramalho de Azevedo Av. das Américas, 1155, sala 301 Rio de Janeiro, RJ, Brasil fernanda@endoclinicbarra.com.br

ORecebido em 14/Mar/2012 Aceito em 21/Ago/2012 


\section{INTRODUÇÃO}

$\mathrm{O}$ iodo radioativo (RAI) é comumente usado como primeira opção terapêutica no tratamento do hipertireoidismo principalmente nos Estados Unidos e em alguns Serviços de Endocrinologia do Brasil. Ele foi empregado pela primeira vez em 1941 no Massachusetts General Hospital por Hertz e Roberts, sendo o isótopo usado na ocasião o $I^{130}(1)$. Em função do baixo custo, da maior eficácia na destruição da célula tireoidiana e da meia-vida de oito dias, $\mathrm{o} \mathrm{I}^{131}$ passou a ser o isótopo de escolha e mantém-se amplamente utilizado até os dias de hoje.

No entanto, sua segurança em longo prazo, principalmente em crianças e adultos jovens, está sendo questionada. Estudos sobre mortalidade em pacientes com hipertireoidismo tratados com RAI são poucos e baseados em três diferentes coortes de pacientes: americanos, suecos e ingleses.

No primeiro estudo realizado por Hoffman e cols., na Mayo Clinic, não foi observada diferença na mortalidade geral entre 1.005 mulheres tratadas com RAI e 2.141 mulheres tratadas com cirurgia como forma de tratamento do hipertireoidismo (2).

Já Goldman e cols. evidenciaram aumento na taxa de mortalidade geral, inclusive por doenças endócrinas, circulatórias e respiratórias, mas não por doenças malignas, em 1.762 mulheres com hipertireoidismo tratadas com RAI (80\%), tireoidectomia ou drogas antitireoidianas, no Massachusetts General Hospital (3).

No estudo sueco realizado por Hall e cols., foi observado que em 10.552 pacientes com hipertireoidismo tratados com RAI houve um aumento significativo na taxa de mortalidade geral, quando comparado com a taxa esperada para a população geral. Nesse estudo, houve risco maior de morte por doenças respiratórias, cardiovasculares e endócrinas, e câncer principalmente dos aparelhos digestivo e respiratório $(4,5)$.

Franklyn e cols. demonstraram em uma coorte de 7.209 pacientes com hipertireoidismo tratados com RAI no Reino Unido que houve aumento em todas as causas de mortalidade, principalmente as secundárias por doença cardiovascular, cerebrovascular, tireoidiana e por fratura de fềmur. No entanto, houve uma redução na mortalidade em decorrência do câncer $(6,7)$.

$\mathrm{O}$ aumento da mortalidade visto em alguns dos estudos citados pode ser reflexo da influência do próprio hipertireoidismo, um efeito adverso específico do RAI, ou ser subsequente ao hipotireoidismo e seu tratamento com tiroxina $(8)$.
O presente trabalho propõe avaliar a taxa de mortalidade e o surgimento de comorbidades cardiovasculares e neoplásicas, como efeito no longo prazo da radioiodoterapia em pacientes tratados para hipertireoidismo em decorrência da doença de Graves há mais de 10 anos.

\section{MATERIAIS E MÉTODOS}

Com o objetivo de avaliar o surgimento de comorbidades cardiovasculares ou neoplásicas e a taxa de mortalidade nos pacientes tratados com iodo radioativo, como forma de tratamento do hipertireoidismo da doença de Graves há mais de 10 anos, foi realizado um estudo retrospectivo com análise de prontuários do Hospital Universitário Clementino Fraga Filho (HUCFF) a partir de dados do Serviço de Medicina Nuclear do hospital.

Foram selecionados os pacientes submetidos à dose terapêutica para tratamento de hipertireoidismo de janeiro de 1981 até novembro de 1999, tendo sido excluídos os pacientes que previamente já tinham o diagnóstico de algumas dessas comorbidades, como hipertensão arterial sistêmica, doença coronariana, ou doença neoplásica já conhecida. Excluíram-se também os pacientes que não tiveram o acompanhamento clínico pós-dose terapêutica por até 10 anos.

Foram também coletados dados sobre outras variáveis que pudessem influenciar no surgimento dessas comorbidades ou na taxa de mortalidade, como idade, tempo de tratamento, número de doses terapêuticas, dose cumulativa e tempo de evolução para o hipotireoidismo, definido como TSH $>4,0 \mathrm{mcU} / \mathrm{mL}$ e T4 livre $<0,8 \mathrm{ng} / \mathrm{dL}$ pelo método de quimioluminescência do kit Immulite 2000.

Para avaliar estatisticamente o surgimento das comorbidades estudadas e a taxa de mortalidade, comparamos nosso grupo de pacientes com um grupo de pacientes eutireoidianos, que foram portadores de hipertireoidismo em função da doença de Graves, mas entraram em remissão clínica e laboratorial após o tratamento com drogas antitireoidianas (DAT) durante o período de agosto de 1988 a setembro de 2002.

O Serviço de Medicina Nuclear do HUCFF calcula a dose de iodo radioativo a ser administrada levando-se em consideração o tamanho da glândula e a captação do iodo por esta, usando uma fórmula padrão [dose ( $\mu \mathrm{Ci}$ de $\mathrm{I}^{131} / \mathrm{g}$ de tireoide $\mathrm{x}$ peso estimado)/captação do radioiodo em $24 \mathrm{~h}]$. 
Os pacientes submetidos à dose terapêutica (DT) eram acompanhados periodicamente no ambulatório, quando submetidos à avaliação clínica e laboratorial. A permanência do estado hipertireoidiano e a necessidade do uso de tionamidas dentro do prazo previsto para a possível evolução para o eutireoidismo, ou até hipotireoidismo, indicavam uma nova administração de iodo radioativo. Já os pacientes que evoluíam para o hipotireoidismo eram prontamente tratados com a reposição de levotiroxina. O mesmo acontecia com o surgimento das comorbidades estudadas, prontamente diagnosticadas e tratadas.

A análise estatística foi composta pelos seguintes métodos: para a comparação de variáveis contínuas (numéricas) entre dois grupos foi utilizado o teste $t$ de Student para amostras independentes ou o teste de Mann-Whitney (não paramétrico). A homogeneidade das variâncias entre os grupos foi analisada pelo teste de Bartlet e, para a comparação de variáveis categóricas (qualitativas) entre dois subgrupos, foi aplicado o teste de Qui-quadrado $\left(\mathrm{X}^{2}\right)$ ou exato de Fisher.

Utilizou-se o método não paramétrico, pois algumas variáveis não apresentaram distribuição normal (distribuição Gaussiana), em decorrência da dispersão dos dados e da falta de simetria da distribuição. O critério de determinação de significância adotado foi o nível de 5\%. A análise estatística foi processada pelo software SAS 6.11 (SAS Institute, Inc., Cary, NC).

\section{RESULTADOS}

A amostra total de prontuários analisados foi de 222. No entanto, 40 (18\%) foram excluídos devido à perda do seguimento clínico ao longo dos 10 anos após a realização da dose terapêutica (DT). Além desses, 75 $(33,7 \%)$ pacientes já apresentavam uma comorbidade que seria objeto de estudo (HAS). Portanto, a amostra total de prontuários incluídos foi de 107 (48,1\%), sendo 93 mulheres e 14 homens.

A tabela 1 demonstra o perfil geral da casuística em relação às variáveis numéricas, como a idade dos pacientes, o tempo de tratamento (da época do diagnóstico até a realização da DT), o número de $\mathrm{DT}$, a dose cumulativa e o tempo de evolução para o hipotireoidismo. A média de idade dos pacientes foi de 54 anos. A mediana da dose de iodo radioativo administrada foi de $12,2 \mathrm{mCi}(451,4 \mathrm{MBq})$.

Os pacientes analisados apresentavam como condições clínicas na época do diagnóstico a presença de
Tabela 1. Descritiva geral das variáveis numéricas

\begin{tabular}{lcccc}
\hline Variável & N & Mediana & Mínimo & Máximo \\
\hline Idade (anos) & 105 & 54,0 & 32 & 85 \\
$\begin{array}{l}\text { Tempo de tratamento } \\
\text { (meses) }\end{array}$ & 83 & 21,0 & 3 & 74 \\
No de DT $^{\text {Dose acumulativa (mCi) }}$ & 107 & 1,0 & 1 & 4 \\
Tempo de evolução & 96 & 12,2 & 4 & 34,5 \\
hipotireoidismo (meses) & & 8,0 & 3 & 132 \\
\hline
\end{tabular}

DT: dose terapêutica.

bócio em $89,7 \%$ destes, oftalmopatia em $38,3 \%$ e o hábito de fumar em $8,4 \%$. A grande maioria dos pacientes, $96,3 \%$, fez tratamento prévio com drogas antitireoidianas antes da dose terapêutica. Tanto a presença de bócio quanto a de oftalmopatia não foram acompanhadas de complicações clínicas pós-DT.

Na tabela 2, observamos que 54 pacientes $(51,4 \%)$ evoluíram ao longo dos 10 anos de acompanhamento após a DT com comorbidades cardiovasculares e/ou neoplásicas. Não houve nenhum óbito. Entre as comorbidades clínicas, a mais frequente foi hipertensão arterial sistêmica (HAS) (29,5\%), seguida pela dislipidemia $(27,6 \%)$. Apenas um caso de doença neoplásica foi registrado. A paciente era do sexo feminino e apresentou câncer de mama. Achamos importante registrar a presença de diabetes melito tipo 2 (DM) em 10,5\% dos pacientes, um fator de risco importante para o agravamento dessas comorbidades.

O hipotireoidismo ocorreu em 96 (89,7\%) pacientes, em um tempo médio de evolução de cerca de 8 meses conforme a tabela 1. Em relação ao surgimento das comorbidades nos pacientes que evoluíram para o hipotireoidismo após a realização da DT, observamos a presença de: HAS em 31 pacientes, dislipidemia em 29, DM em 11 , doença coronariana em 5 e câncer em 1 paciente. Já entre os pacientes que evoluíram para o eu-

Tabela 2. Descritiva geral das comorbidades

\begin{tabular}{lcc}
\hline Variável & $\mathbf{n}$ & \% \\
\hline Comorbidade geral $^{*}$ & 54 & 51,4 \\
HAS $^{*}$ & 31 & 29,5 \\
Dislipidemia* $^{*}$ & 29 & 27,6 \\
Doença coronariana* $^{*}$ & 5 & 4,8 \\
DM* $^{*}$ & 11 & 10,5 \\
Câncer* $^{*}$ & 1 & 1,0 \\
AVC $^{*}$ & 1 & 1,0 \\
Óbito* & 0 & 0 \\
\hline
\end{tabular}

* Perda de dois pacientes. AVC: acidente vascular cerebral; DM: diabetes melito; HAS: hipertensão arterial sistêmica. 
tireoidismo após a DT, 4 apresentaram HAS (12,9\%), 1 apresentou dislipidemia $(3,4 \%)$ e 1 , AVC (1\%), ao longo dos 10 anos de acompanhamento.

Comparamos o surgimento dessas comorbidades com um grupo controle formado por pacientes portadores de hipertireoidismo em decorrência da doença de Graves que entraram em remissão clínica e laboratorial após o tratamento com drogas antitireoidianas (DAT). O grupo controle é formado por 30 pacientes, com uma média de idade de 49,5 anos, tratados com uma DAT por um tempo médio de 15,7 meses.

Como vemos na tabela 3 , nenhum paciente do grupo controle apresentou as comorbidades avaliadas no estudo. Comparando o grupo de pacientes tratados com iodo radiativo e o grupo de pacientes tratados com DAT, o surgimento de HAS e dislipidemia foi estatisticamente significativo no grupo de pacientes tratados com iodo radiativo.

$\mathrm{Na}$ amostra, $72 \%$ dos pacientes receberam apenas 1 DT. Os pacientes foram divididos em dois subgrupos, um grupo que recebeu apenas 1 dose terapêutica (DT) $(\mathrm{DT}=1)$ e outro grupo que recebeu mais de $\mathrm{IDT}$ (DT $>1$ ). Foram comparados esses dois grupos, número de $\mathrm{DT}=\mathrm{l}$ e $>1$, em relação a idade, tempo de tratamento, dose cumulativa e tempo de evolução para o hipotireoidismo, para verificar se existia uma diferença significativa entre essas variáveis. Observou-se que o subgrupo com número de DT $>1$ apenas apresentou tempo de tratamento $(\mathrm{p}=0,001)$ e dose cumulativa $(\mathrm{p}=0,0001)$ significativamente maior que o subgrupo com número de DT $=1$. Não observamos que o grupo de DT > 1 tenha evoluído mais rápido para o hipotireoidismo.

Tabela 3. Comparação no surgimento de comorbidades

\begin{tabular}{|c|c|c|c|c|c|}
\hline \multirow{2}{*}{ Variável } & \multicolumn{2}{|c|}{$\begin{array}{l}\text { lodo radiativo } \\
(\mathrm{n}=\mathbf{1 0 7})\end{array}$} & \multicolumn{2}{|c|}{ DAT $(n=30)$} & \multirow{2}{*}{ p valor ${ }^{a}$} \\
\hline & $\mathbf{n}$ & $\%$ & $\mathbf{n}$ & $\%$ & \\
\hline Comorbidade geral & 54 & 50,5 & 0 & 0 & $<0,0001$ \\
\hline $\mathrm{HAS}^{*}$ & 31 & 29,0 & 0 & 0 & 0,001 \\
\hline Dislipidemia** & 29 & 27,1 & 0 & 0 & 0,002 \\
\hline Doença coronariana & 5 & 4,7 & 0 & 0 & 0,28 \\
\hline DM & 11 & 10,3 & 0 & 0 & 0,058 \\
\hline Câncer & 1 & 0,93 & 0 & 0 & 0,78 \\
\hline AVC & 1 & 0,93 & 0 & 0 & 0,78 \\
\hline Óbito & 0 & 0 & 0 & 0 & NSA \\
\hline
\end{tabular}

* Perda de 3 pacientes no grupo DAT. ** Perda de 1 paciente no grupo DAT.

a Teste de $\chi^{2}$ ou exato de Fisher.

AVC: acidente vascular cerebral; DAT: drogas antitireoidianas; DM: diabetes melito; HAS: hipertensão arterial sistêmica.
Comparando a idade, o tempo de tratamento, a dose cumulativa e o tempo de evolução para o hipotireoidismo, com a presença ou não de comorbidades, observou-se que o subgrupo com comorbidades apresentou apenas a idade $(\mathrm{p}=0,001)$ significativamente maior que o subgrupo sem comorbidades. Logo, o tempo de tratamento, o tempo de evolução para o hipotireoidismo e a dose cumulativa não influenciaram no surgimento das comorbidades em geral e separadamente.

\section{DISCUSSÃO}

O iodo radioativo está sendo cada vez mais utilizado como forma de tratamento do hipertireoidismo em nosso país. Neste estudo, avaliamos o surgimento de comorbidades cardiovasculares e neoplásicas e a taxa de mortalidade nos pacientes que receberam o iodo radioativo há mais de 10 anos.

Observamos um aumento estatisticamente significativo no surgimento de HAS e dislipidemia quando se compara com um grupo controle de pacientes eutireoidianos, acompanhados pelo Serviço de Endocrinologia do HUCFF, que foram portadores de hipertireoidismo devido à doença de Graves e tratados com DAT.

Este grupo de pacientes apresenta uma média de idade de 49,5 anos, menor do que a do grupo de pacientes tratados com iodo radiativo, que é de 54 anos. O tempo médio de tratamento do grupo de pacientes eutireoidianos, 15,7 meses, também foi inferior ao tempo de tratamento do grupo de pacientes que recebeu iodo radioativo. Logo, a idade e o tempo de exposição ao hipertireoidismo foram maiores no grupo de pacientes tratados com iodo radioativo.

Mesmo com um aumento significativo no surgimento de HAS e dislipidemia, não observamos aumento na taxa de mortalidade.

Esse resultado é discordante da maioria dos estudos que encontraram uma maior mortalidade nos pacientes tratados com iodo radioativo quando comparados com a população geral. Diferente desses estudos (2-9), foram avaliados apenas os pacientes tratados em decorrência da doença de Graves e excluídos os que já apresentavam as comorbidades avaliadas pelo estudo. A média de idade neste trabalho foi de 54 anos, mais baixa que dos outros estudos (média de 62 anos) (10).

Além disso, o tempo de observação em alguns estudos também ultrapassou os 20 anos, como o estudo de coorte de Franklyn e cols. (7), que avaliou a incidência de câncer e mortalidade após a terapêutica com iodo radioativo para o hipertireoidismo por 40 anos. 
O estudo de Metso e cols. (10) tinha como objetivo comparar a taxa de mortalidade dos pacientes portadores de hipertireoidismo que foram tratados com iodo radioativo entre 1965 e 2002 com a taxa da população geral. Observaram-se cerca de 2.790 pacientes, dos quais $57 \%$ tinham doença de Graves e $43 \%$, doença nodular da tireoide (bócio multinodular e adenoma tóxico). A mortalidade foi mais elevada nos pacientes com doença nodular e não nos pacientes com doença de Graves quando comparados com os controles. Além disso, o risco de morte foi maior com o aumento da dose cumulativa do iodo radioativo e com a idade. A média de acompanhamento foi de 9 anos para os pacientes e de 9,4 anos para os controles. No entanto, o risco de morte foi mais elevado nos pacientes tratados com iodo radioativo quando comparados com os controles, quando o tempo de acompanhamento era maior do que 25 anos.

Os estudos que definiram que o risco de morte devido a um evento cardiovascular é mais frequente nos pacientes tratados com iodo radioativo, quando comparados com seus controles, não souberam definir se esse risco de mortalidade cardiovascular está relacionado ao tratamento com iodo radioativo ou se é decorrente do próprio efeito do hipertireoidismo, hipótese mais provável $(3,4,6,8,10)$. Essa distinção entre os efeitos do tratamento com os da doença só seria possível se tivéssemos um grupo de pacientes não tratados como referência.

Somente no estudo de Franklyn e cols. (6) os resultados evidenciaram maior prevalência de doenças cardiovasculares como causa do aumento da taxa de mortalidade. Nesse estudo, a doença cerebrovascular e a coronariana foram as que determinaram esse aumento da mortalidade. De um total de 7.209 pacientes, 3.611 morreram durante o acompanhamento, sendo que o número de morte esperado era de 3.186. Em relação às doenças cardiovasculares, o excesso de morte foi de 240 a mais do que o esperado. Além disso, observaram que o aumento da mortalidade foi mais evidente no primeiro ano após o tratamento com o iodo radioativo e que foi se reduzindo com o tempo.

O hipertireoidismo tem efeito direto sobre o miocárdio e o sistema nervoso autônomo, podendo predispor o paciente a arritmias, principalmente a fibrilação atrial $(11,12)$. Flyn e cols. (9) reportaram um aumento no risco de arritmia mesmo após cerca de cinco anos de tratamento do hipertireoidismo, sugerindo que os efeitos cardiotóxicos do hipertireoidismo não são com- pletamente interrompidos com a restauração do eutireoidismo.

Resultados sobre a mortalidade de doenças neoplásicas nos pacientes tratados com iodo radioativo são conflitantes. Há relatos de serem maiores $(4,13)$, menores $(7)$ e iguais $(3,14)$ quando comparados com a população geral. A dose total de iodo radioativo não foi significantemente diferente no estudo que demonstrou redução na taxa de mortalidade por doença neoplásica, quando comparada com a dos estudos que mostram aumento nessa taxa. No entanto, os pacientes no estudo de Metso e cols. (10), em que se observou aumento da mortalidade, eram um pouco mais idosos do que no estudo de Franklyn e cols. (7).

O trabalho de Metso e cols. (10) confirma os resultados de Hall e cols. (4), que observaram que o iodo radioativo pode contribuir para o aumento da mortalidade devido ao câncer de estômago, visto no estudo. Quando se utiliza o iodo radioativo como tratamento para o hipertireoidismo, a dose da radiação para os tecidos não tireoidianos é relativamente baixa (menos de $10 \mathrm{cGy}$ ), exceto para os órgãos que acumulam o iodo, como o estômago $(25 \mathrm{cGy})(15,16)$. Consequentemente, o estômago pode estar particularmente vulnerável à indução de câncer pela radiação.

No estudo em questão, só tivemos um caso registrado de doença neoplásica (câncer de mama). No entanto, como já discutido, o tempo de observação para avaliar o surgimento de tumor, principalmente de tumores sólidos, deve ser maior.

Segundo o guideline da American Thyroid Association (ATA) (17), recomenda-se que o tratamento com iodo radioativo seja evitado em crianças menores que 5 anos e avaliado de forma criteriosa nas crianças com idade entre 5 e 10 anos de idade. É enfatizado que essas considerações são baseadas em riscos teóricos de desenvolvimento de câncer na tireoide e de leucemia, e que mais estudos sobre esses assuntos são necessários.

Esses resultados conflitantes sobre a taxa de mortalidade de doenças neoplásicas após o tratamento do hipertireoidismo com iodo radioativo podem ser influenciados por fatores que podem mudar a sensibilidade individual à radiação, como a idade à época da exposição, o hábito de fumar e a dieta.

O hipotireoidismo também foi sugerido como um fator contribuinte no elevado risco de morte devido aos efeitos que pode causar, como a hipercolesterolemia a hipertensão diastólica e a disfunção do ventrículo esquerdo (18). A maioria dos estudos perdeu o registro 
do início do desenvolvimento do hipotireoidismo. No nosso estudo, assim como o de Metso, o tratamento com levotiroxina pareceu proteger contra esse aumento de morte em vez de predispor. E o tempo médio de evolução para o hipotireoidismo foi de 8 meses.

Vários fatores podem influenciar o surgimento das comorbidades cardiovasculares e neoplásicas, como fatores ambientais e hereditários. No estudo apresentado, o tratamento com iodo radioativo nos pacientes portadores de hipertireoidismo causado pela doença de Graves acarretou o surgimento de forma estatisticamente significativa de HAS e dislipidemia, quando comparado com um grupo de pacientes eutireoidianos após uso de DAT para tratamento do hipertireoidismo causado pela doença de Graves. Não foi observado aumento da taxa de mortalidade.

Em conclusão, acreditamos que é necessário um tempo maior de acompanhamento para avaliar a real influência da terapêutica com iodo radioativo no surgimento dessas comorbidades e na taxa de mortalidade. A idade e o tempo de exposição aos efeitos do hipertireoidismo parecem influenciar no surgimento dessas comorbidades.

Declaração: os autores declaram não haver conflitos de interesse científico neste estudo.

\section{REFERÊNCIAS}

1. Sawin CT, Becker DV. Radioiodine and the treatment of hyperthyroidism: the early history. Thyroid. 1997;7:163-76.

2. Hoffman DA, McConhey WM, Diamond EL, Kurland LT. Mortality in women treated for hyperthyroidism. Am J Epidemiol. 1982;115:243-54.

3. Goldman MB, Maloof F, Monson RR, Aschengrau A, Cooper DS, Ridgway EC. Radioactive iodine therapy and breast cancer.
A follow up study of hyperthyroid women. Am J Epidemiol. 1988;127:969-80.

4. Hall P, Berg G, Bjelkengren G, Boice Jr JD, Ericsson UB, Hallquist A, et al. Cancer mortality after iodine-131 therapy for hyperthyroidism. Int J Cancer. 1992;50:886-90.

5. Hall P, Lendell G, Holm LE. Mortality in patients treated for hyperthyroidism with iodine-131. Acta Endocrinol. 1993;128:230-4.

6. Franklyn JA, Maisonneuve P, Sheppard M, Betteridge J, Boyle P. Mortality after the treatment of hyperthyroidism with radioactive iodine. N Engl J Med. 1998;338:712-8.

7. Franklyn JA, Maisonneuve P, Sheppard M, Betteridge J, Boyle P. Cancer incidence and mortality after radioiodine treatment for hyperthyroidism: a populacion based cohort study. Lancet. 1991;353:2111-5.

8. Franklyn JA, Sheppard MC, Maisonneuve P. Thyroid function and mortality in patients treated for hyperthyroidism. JAMA. 2005;294:71-80.

9. Flynn RW, MacdonaldTM, Jung RT, Morris AD, Leese GP. Mortality and vascular outcomes in patients treated for thyroid dysfunction. J Clin Endocrinol Metab. 2006;91:2159-64.

10. Metso S, Jaatinen $P$, Huhtala $H$, Auvinen A, Oksala H, Salmi J. Increase cardiovascular and cancer mortality after radioiodine treatment for hiperthyroidism. J Clin Endocrinol Metab. 2007;92(6):2033-5.

11. Osman F, Gammage MD, Sheppard MC, Franklyn JA. Clinical review 142: cardiac dysrhythmias and thyroid dysfunction: the hidden menace? J Clin Endocrinol Metab. 2002;87:963-7.

12. Osman F, Gammage MD, Franklyn JA. Hyperthyroidism and cardiovascular morbidity and mortality. Thyroid. 2002;12:483-7.

13. Goldman MB, Monson RR, Maloof F. Cancer mortality in women with thyroid disease. Cancer Res. 1990;50:2283-9.

14. Ron E, Doody MM, Becker DV, Brill AB, et al. Cancer mortality following treatment for adult hyperthyroidism. JAMA. 1998;280:347-55.

15. Holm LE, Hall P, Wiklund K, Lundell G, et al. Cancer risk after iodine-131 therapy for hyperthyroidism. J Natl Cancer Inst. 1991;83:1072-7.

16. Edmonds $\mathrm{CJ}$, Smith $\mathrm{T}$. The long-term hazards of the treatment of thyroid cancer with radioiodine. Br J Radiol. 1986;59:45-51.

17. Bahn RS, Burch HB, Cooper DS, Garber, JR, Greenlee MC, Klein I, et al. Hyperthyroidism and oher causes of thyrotoxicosis: management guidelines of the American Thyroid Association and American Association of Clinical Endocrinologists. Thyroid. 2011;21:593-641.

18. Cappola AR, Landenson PW. Hypothyroidism and atherosclerosis. J Clin Endocrinol Metab. 2003;88:2438-44. 